\title{
Abortamento Induzido: vivência de mulheres baianas
}

\section{Induced Abortion: the experience of women from the Brazilian State of Bahia}

\author{
Vanessa do Nascimento Pereira \\ Enfermeira Assistencial do Hospital Regional de Santo Antônio de \\ Jesus e do Conjunto Penal de Valença. \\ Endereço: Campus Universitário do Canela. Av. Dr. Augusto Viana \\ S/N, 70 andar. Canela, CEP 40110-060, Salvador, Bahia, Brasil. \\ E-mail: nessinha_fjøhotmail.com \\ Flávia Aelo de Oliveira \\ Enfermeira Residente em UTI pela Universidade Federal da \\ Bahia. \\ Endereço: Campus Universitário do Canela. Av. Dr. Augusto Viana \\ S/N, 70 andar, CEP 40110-060, Canela, Salvador, Bahia, Brasil. \\ E-mail: flaviaaeloळhotmail.com

\section{Nadirlene Pereira Gomes} \\ Doutora em Enfermagem. Professora Adjunta da Escola de Enfer- \\ magem da Universidade Federal da Bahia. \\ Endereço: Campus Universitário do Canela. Av. Dr. Augusto Viana \\ S/N, 70 andar. Canela, CEP 40110-060, Salvador, Bahia, Brasil. \\ E-mail: nadirlenegomesळhotmail.com

\section{Telmara Menezes Couto} \\ Doutora em Enfermagem. Professora Adjunta da Universidade \\ Federal da Bahia. \\ Endereço: Campus Universitário do Canela. Av. Dr. Augusto Viana \\ S/N, 70 andar. Canela, CEP 40110-060, Salvador, Bahia Brasil. \\ E-mail: telmaracouto®gmail.com

\section{Gilvânia Patrícia do Nascimento Paixão} \\ Enfermeira Obstetra, mestranda em Enfermagem pela Universi- \\ dade Federal da Bahia. Professora Auxiliar da Universidade do \\ Estado da Bahia. \\ Endereço: Rua Jogo do Carneiro, 352, Bloco II, Ap. 105, Saúde, CEP \\ 40045-040. Salvador, Bahia, Brasil. \\ E-mail: gilvania.paixao®gmail.com
}

\section{Resumo}

O abortamento representa grave problema de saúde pública, que envolve questões legais, econômicas, sociais e psicológicas. Estudo qualitativo que teve como objetivo identificar situações que interferem na decisão pelo aborto e os sentimentos diante do processo de abortar. 0 estudo foi realizado em uma Maternidade Pública, no Município de Salvador-BA, e os sujeitos foram constituídos por nove mulheres hospitalizadas por aborto provocado. Para a coleta de dados, utilizamos a entrevista acompanhada por um formulário semiestruturado. Foram considerados os aspectos éticos baseados na Resolução 196/96 do Conselho Nacional de Saúde. Para a análise das falas, usamos como referencial a técnica de análise de conteúdo de Bardin. A amostra caracterizou-se por mulheres adultas eminentemente negras; casadas/união estável; dependentes financeiramente do companheiro. Na análise dos discursos, surgiram dois temas: Motivação e Sentimentos. Dentre os motivos que levam ao aborto, encontram-se a dificuldade financeira, o número de filhos, a vivência de violência conjugal e a perda de sua autonomia. 0 processo de abortamento gera medo de morrer, tristeza e alívio. As mulheres vivenciam o aborto induzido, revelando um processo muito doloroso, desde o momento em que descobrem a gravidez, passando pela difícil decisão de interrompê-la. Quando não são ajudadas, essas mulheres perpetuam essa dor, vivendo dias de angústia e culpa. 0 exercício da escuta e do acolhimento devem estar presentes na vida dos profissionais de saúde, independentemente de suas opiniões com relação ao aborto, a fim de que a mulher possa expressar seus sentimentos, e então obter ajuda e encaminhamento adequados.

Palavras-chave: Saúde da mulher; Aborto induzido; Políticas Públicas. 


\section{Abstract}

Abortion is a serious public health problem that involves legal, economic, social and psychological issues. This qualitative study aimed to identify situations that interfere in the abortion decision and the feelings about the process of aborting. The study was conducted in a public maternity hospital in the city of Salvador (Northeastern Brazil), and the subjects were nine women hospitalized due to induced abortion. To collect data, we used the interview accompanied by a semi-structured form. Ethical aspects were considered based on Resolution 196/96 of the National Health Council. For the analysis of the discourses, we used Bardin's content analysis technique. The sample was characterized by predominantly black adult women, married or in a stable union, financially dependent on the partner. In the analysis, two themes emerged: motivation and feelings. Among the reasons leading to abortion, there are financial difficulties, the number of children, the experience of domestic violence and the loss of their autonomy. The process of aborting generates fear of death, sadness and relief. Women experiencing induced abortion revealed a very painful process from the moment they discover the pregnancy until the difficult decision to interrupt it. When they are not helped, these women perpetuate the pain, living days of anguish and guilt. The exercise of listening and welcoming these women must be present in the lives of health professionals, regardless of their views on abortion, so that the women can express their feelings, and then get appropriate help and referral.

Keywords: Women's Health; Induced Abortion; Public Policy.

\section{Introdução}

O enorme número de gestações interrompidas é uma questão de saúde pública, devido às repercussões na saúde da mulher e aos gastos com internações e procedimentos hospitalares, já que o abortamento representa uma das principais causas de morte materna no Brasil e a curetagem, o segundo procedimento obstétrico mais realizado nas unidades de internação da rede pública de serviços de saúde, superada apenas pelos partos normais (Brasil, 2005).

Segundo o Serviço de Informações Hospitalares do Sistema Único de Saúde, estima-se que em 2005 foram realizados 1.054.242 abortamentos, números abaixo da realidade devido à subnotificação. Isso se justifica pelo fato de as mulheres sentirem-se geralmente constrangidas ou amedrontadas para declarar o procedimento nos serviços de saúde (Brasil, 2009).

Diante do medo, a interrupção da gravidez é feita clandestinamente, sendo que, para as mulheres com poucos recursos financeiros, a prática é insegura e sob péssimas condições (Scavone e Cortes, 200o). Segundo a Organização Mundial de Saúde (1999), estima-se que 70 a 80 mil mulheres morrem por conta do abortamento inseguro, e que $95 \%$ destes sejam realizados em países em desenvolvimento. Acredita-se ainda que cerca de $20 \%$ das mortes maternas em todo o mundo sejam resultados das práticas ilegais e, portanto, inseguras.

Nesta perspectiva, o conhecimento e a compreensão dos motivos que levam à decisão pela interrupção da gravidez são subsídios para intervenção nos problemas de ordem social, que muitas vezes subjazem a motivação da mulher para optar pelo aborto.

Além disso, a reflexão acerca desse problema pode levar o profissional a reorientar a sua conduta, prestando uma assistência livre de julgamentos pessoais.

Frente ao exposto, o estudo tem como objetivo identificar situações que interferem na decisão pelo aborto e os sentimentos diante do processo de abortar. 


\section{Métodos}

Trata-se de uma pesquisa com abordagem qualitativa, de cunho descritivo. 0 estudo foi realizado em uma Maternidade Pública, no Município de Salvador, capital do Estado da Bahia, e os sujeitos foram constituídos por nove mulheres hospitalizadas por aborto provocado.

Os dados foram coletados nos meses de setembro e outubro de 2009, de segunda a sexta-feira, no período da tarde. Consideramos como critério de inclusão a idade gestacional de até vinte e duas semanas na ocasião do aborto, a autodeclaração de ter induzido o aborto e a maioridade. Portanto, as mulheres menores de 18 anos foram excluídas deste estudo, pois, para fazer pesquisa com sujeitos desta faixa etária, é necessário um representante legal para consentir a coleta de dados, conforme a Resolução 196/96 (Brasil,1996). Estando as mulheres deste estudo em situação de prática ilegal, a necessidade de um representante para consentimento dificultaria a pesquisa. Com a idade adulta, apenas uma mulher não aceitou participar do estudo, totalizando a amostra de nove mulheres.

Como técnica para coleta de dados, foi utilizada a entrevista. Para tanto, elaborou-se um formulário semiestruturado como instrumento de coleta de dados, que consta de três partes: (1) dados sociodemográficos, (2) dados gineco-obstétricos, e (3) motivos para o abortamento. $\mathrm{O}$ estudo foi aprovado pelo Comitê de Ética da Secretaria do Estado da Bahia Parecer no 334/2008 Registro no CEP: CAAE 0110.0.053.053-08 sendo, portanto, garantido o sigilo das informações e o caráter voluntário da participação, sem prejuízo da assistência na maternidade. As mulheres foram identificadas por nome de flores para garantir o anonimato.

As falas foram organizadas e categorizadas, utilizando como referencial a técnica de analise de conteúdo de Bardin. A categorização é um processo composto de duas etapas: o inventário e a classificação (Bardin, 2009). Nestas etapas, isolamos as falas das mulheres e então procuramos organizar as falas dentro das categorias estabelecidas, a fim de se obter uma organização das mensagens.

Após leitura exaustiva das falas, foram construídos dois temas e nove categorias, as quais re- presentam a síntese do conteúdo das entrevistas e tiveram inicialmente como foco o agrupamento das motivações para a interrupção da gravidez. Para o tema "Motivação" foram construídas as categorias: dificuldade financeira, número de filhos, vivência de violência conjugal, perda da autonomia. Enquanto que para o tema "Sentimentos" foram definidas como categorias: medo de morrer, tristeza e alívio.

\section{Resultados e Discussão}

\section{Caracterização dos sujeitos}

No que tange à caracterização das participantes, a amostra foi composta majoritariamente por mulheres entre 20 e 29 anos (66,6\%), portanto adultas jovens. Estudos relatam que a faixa etária mais encontrada nos casos de abortos é entre 20 e 29 anos. (Cecatti e col., 2010; Silva e col., 2010; Ramos e col., 2010).

Quanto à etnia, esta foi autodeclarada, totalizando $77,7 \%$ de mulheres da raça negra. Um estudo realizado por Barbosa (2009) evidenciou que as mulheres que autodeclararam ser preta ou parda tiveram proporção significativamente maior de abortos induzidos em relação às mulheres brancas.

Observamos que a maioria possui nenhum ou poucos anos de estudo (77,7\%); a maioria vive em união consensual ou casamento civil (66,6\%); todas têm pelo menos um filho. Dados semelhantes foram revelados em estudo realizado por Silva e colaboradores (2010) que percebeu que a escolaridade das mulheres que induziram o aborto era de até o segundo grau (57,2\%), a maioria tinha companheiro fixo $(61 \%)$ e dois terços referiram gestações anteriores $(67,2 \%)$.

A maioria das entrevistadas depende economicamente do companheiro ou dos pais $(66,6 \%)$. Este achado aproxima-se do resultado encontrado por Ramos e colaboradores (2010) em que 50\% das mulheres que induziram aborto estavam desempregadas.

Quase que na totalidade $(88,9 \%)$ declararam ter realizado o aborto pela primeira vez. Estudo de Pérez (2006) também mostrou que a maioria das mulheres $(64,6 \%)$ declararam estar praticando aborto pela primeira vez. 
No que tange à idade gestacional na ocasião do aborto, $77,7 \%$ das entrevistadas referiram ter interrompido a gravidez no $1^{\circ}$ - trimestre, e, $88,8 \%$ citaram o uso do misoprostol como método abortivo. O Ministério da Saúde evidenciou o uso deste método em quase $85 \%$ das mulheres que induziram o aborto (Brasil, 2009).

Todas as entrevistadas referiram estar em uso de algum método anticoncepcional quando descobriram a gestação, sendo o anticoncepcional hormonal (oral ou injetável) o mais utilizado por elas. Pesquisas realizadas com mulheres em maternidades públicas evidenciam que a maior parcela delas conhece os contraceptivos, sobretudo pílula e preservativo, embora o conhecimento sobre concepção e contracepção seja frágil, inconsistente, explicando em parte o uso pouco efetivo dos contraceptivos (Almeida e col., 2003). Logo, percebe-se que o conhecimento limitado sobre o uso de contraceptivos e a falta de programas de atenção à contracepção influenciam o uso inadequado, abandono e falha do método, o que pode favorecer o abortamento inseguro (Silva e Vieira, 2009).

O fato de a maioria das mulheres serem negras, ter baixa escolaridade e depender financeiramente de alguém (geralmente do companheiro), pode ter relação com a característica do lócus da pesquisa, uma vez que há um predomínio da clientela de baixo poder aquisitivo nas instituições públicas.

\section{Tema: motivação}

$\mathrm{Na}$ análise das falas, o tema motivação dispõe acerca de situações que interferem na decisão da mulher pela interrupção da gravidez. Embora haja a fragmentação destas situações em categorias, com fins didáticos e analíticos, a decisão de abortar é baseada em uma complexa rede de motivos, e não apenas em um.

A dificuldade financeira é um fator que está associado à decisão de abortar. Além disso, é acentuado por muitos outros fatores, como número de filhos, a dependência financeira (parcial ou total) de algum provedor, nível de escolaridade, o acesso aos métodos contraceptivos, dentre outros. Neste trabalho, a dificuldade financeira da família, como motivo para interromper a gestação, foi mencionada por quatro mulheres $(44,4 \%)$, como na fala a seguir:

Eu não tenho condição... eu vendo água na rua, faço faxina... unha... e consigo uns 50 reais no mês... (Flor-de-Lis).

Meu marido está preso... preso por tráfico de drogas... se ele estivesse aqui fora..." (Íris)

Esse resultado está de acordo com Nader e colaboradores (2007) quando refere que a condição financeira desfavorável está diretamente relacionada com a decisão de abortar.

No tocante ao número de filhos, seis mulheres $(66,6 \%)$ relataram que este fator influenciou na decisão de interromper a gravidez.

Já tenho dois filhos... não tem como criar outro" (Acácia).

Não quero mais filho... mais não... é filho demais! Pra que tanto filho? (Margarida).

O Ministério da Saúde, em estudo sobre o aborto no Brasil, revelou que apenas entre 9,5\% e 29,2\% de todas as mulheres que abortam não tem filhos, um dado que leva muitos estudos a inferir que o aborto é um instrumento de planejamento reprodutivo importante para as mulheres com filhos quando os métodos contraceptivos falham, não são utilizados adequadamente ou quando a assistência prestada pelos profissionais não contempla todas as necessidades da mulher, seja em nível de informação, seja de acompanhamento como previsto pelo Ministério da Saúde (Brasil, 2009).

0 estudo também mostra que a vivência de violência conjugal é um dos elementos que interferem na decisão da mulher pelo aborto.

...a gente não tá se dando muito bem... ele me grita, já me bateu duas vezes... imagine mais um filho nessa situação? (Angélica)

A violência é um dos motivos preponderantes para justificar a interrupção da gravidez (Pérez, 2006). Em consonância, Gesteira (2006) e Sandi e Braz (2010) apresentam a violência doméstica como fator decisivo na interrupção da gravidez.

Contudo, a vivência de violência pode se manifestar pela falta de apoio, desprezo ou pela negação da paternidade. Constitui ainda um fator de risco para o sofrimento psíquico e depressão após o aborto (Mariutti e Furegato, 2010). 
Ele me disse: faça (o aborto), que vai ser melhor! Vai ser melhor pra mim, pra você...vai ser melhor pra nós dois... (Flor-de-Lis)

“... quando ele soube da gravidez, ele mudou... antes ele me ligava todo dia, ia lá no trabalho me ver... eu fiquei achando estranho...fiquei decepcionada. (Hortência).

...ele disse que o filho não era dele... não queria a gravidez... (Açucena)

A decisão do abortamento costuma ser atribuída unicamente às mulheres, negligenciando-se a influência de outros atores, principalmente no que diz respeito ao companheiro. A lei brasileira diz que o aborto é crime para a pessoa ou mulher que o comete, mas não menciona punição ao companheiro que coage a mulher a fazê-lo, e isento de responsabilidades, ele influencia na decisão de abortar (Boemer e Mariutti, 2003; Nader e col., 2007). Além disso, sentindo-se incapazes de assumir sozinhas a criação do filho, as mulheres decidem pela interrupção da gestação (Chumpitaz, 2003).

É importante considerar que a gravidez e um futuro filho desencadeiam as modificações do estilo de vida, o que pode levar à perda da autonomia da mulher. No entanto, esta categoria encontra-se imbricada com as outras: dificuldade financeira, número de filhos e vivência de violência conjugal.

Com um filho dele, e com um emprego pouco seguro, eu podia ficar dependente... isso eu não quero! (Amarilis).

Não queria deixar o trabalho... não podia ficar sem trabalhar. (Hortência).

A gravidez, para essas mulheres, maximiza a subordinação e a dependência financeira, adiando ainda mais a sonhada liberdade. Assim, o aborto se apresenta como um momento de autonomia, possibilitando à mulher a realização de seus planos de vida (Souza, 200o).

\section{Tema: sentimentos}

As falas revelam que o processo de abortar é permeado por uma diversidade de sentimentos que se apresentam de forma desencadeada desde a descoberta da gravidez, passando pelo período que envolve a decisão pela interrupção e que tendem a perdurar mesmo após a consumação do aborto. Este tema "Sentimentos" agrega as seguintes categorias: medo de morrer, tristeza e alívio.

0 estudo mostra que a complexidade que envolve a decisão pela interrupção da gravidez e o processo de abortar tornam ainda mais difícil a ação para o aborto.

Eu fiquei com dúvida de colocar ou não... fiquei com ele (cytotec) na mão só olhando... (Amarilis).

Geralmente, sozinha, a mulher decide pelo aborto e, sozinha, ela o realiza. É a própria mulher quem administra a medicação. É ela quem "coloca o dedo no gatilho”. Todavia, há uma hesitação quanto ao desejo de abortar, a decisão de tomar ou não a medicação, e o medo de morrer (Pérez, 20o6).

Um dos sentimentos mais aludidos ao processo de abortar foi, justamente, o medo de morrer. As falas trazem este sentimento diante da vivência real do aborto:

... quando eu tava sangrando, me deu medo de morrer... eu achei que ia morrer mesmo (Amarílis)

Eu vou morrer... Eu estou arrependida... Deus vaime castigar... eu tirei uma vida (chora) (Flor-de-Liz)

Ao optar e proceder ao abortamento, a mulher vivencia, além do medo de morrer, uma variedade de outros sentimentos como o arrependimento. 0 medo de morrer existe pelo fato de a mulher conhecer os riscos do aborto induzido e, no entanto, a crença no castigo divino intensifica o medo da morte (Benute e col., 2009).

Assim, quando decidem interromper a gravidez, elas se encontram em conflito em virtude de suas crenças, princípios religiosos e valores, os quais contribuem para o aparecimento do sentimento de culpa, culminando no arrependimento (Gesteira e col., 2006).

As falas sinalizam para o sentimento de ambivalência por parte das mulheres após realizarem o aborto: alívio, diante do sucesso do aborto e do fato de estar viva, e tristeza por ter "tirado uma vida".

Agora tô confusa... bate um alívio de tá viva e um arrependimento também (chora) (Amarilis)

... sempre fica alguma coisa na consciência (Margarida) 
Não me arrependo, mas tô triste, afinal é uma vida (chora)... E eu tirei essa vida... mas tô sentindo alívio por não ter morrido (Jasmim)

O alívio é um sentimento frequentemente omitido pelo medo do julgamento do outro, fazendo com que a mulher se coloque numa posição de isolamento e silêncio (Pérez, 2006). Os sentimentos de tristeza e culpa, evidenciados em muitas falas, constituem importante fator de risco para depressão. Segundo um estudo realizado em São Paulo com 50 mulheres, em que se aplicou a escala HAd (Hospital Anxiety and Depression), desenvolvida para pacientes não psiquiátricos, $16 \%$ delas desenvolveram depressão após o aborto induzido (Benute e col., 2009). Para evitar tais situações, faz-se necessária uma assistência livre de julgamentos e permeada pela empatia e acolhimento, centrada não apenas nos aspectos físicos, mas também nos emocionais, a fim de se estabelecer uma relação de confiança.

\section{Considerações}

Os discursos mostram como as mulheres vivenciam o aborto induzido, revelando um processo muito doloroso, desde o momento em que descobrem a gravidez, passando pela difícil decisão de interrompê-la. Quando não são ajudadas, essas mulheres perpetuam essa dor, vivendo dias de angústia, culpa, podendo levar à depressão.

Acredita-se que a tomada de conhecimento sobre os motivos para o aborto induzido, de uma perspectiva mais humanizada, possa contribuir para a efetivação de políticas públicas, já existentes, de assistência à mulher em abortamento.

Para os profissionais de saúde, é essencial saber lidar com as mulheres em abortamento, a fim de estabelecer uma relação não somente do ponto de vista técnico, mas que demonstre interesse, afeto, respeito e empatia, contribuindo para um melhor enfrentamento desse momento difícil.

Os exercícios da escuta e do acolhimento devem estar presentes na vida dos profissionais de saúde, independentemente de suas opiniões com relação ao aborto, a fim de que a mulher possa expressar seus sentimentos, e então obter ajuda e encaminhamento adequados. A própria escuta e o acolhimento são maneiras de evitar que esses sentimentos que permeiam a opção de abortar perdurem, causando malefícios à saúde mental da mulher.

Consideramos que o aborto provocado constituise em um fator de risco para o adoecimento físico e psicológico dessas mulheres, uma vez que sua realização se dá de maneira dolorosa, levando a consequências traumáticas. Apesar de grandes avanços nas discussões e estudos sobre o aborto provocado, é necessário ampliar ainda mais as reflexões sobre essa temática, a fim de melhorar a atenção à saúde das mulheres que decidem pelo aborto.

\section{Referências}

ALMEIDA, M. C. C. et al. Uso de contracepção por adolescentes de escolas públicas na Bahia. Revista de Saúde Pública, São Paulo, v. 37, n. 5, p. 566-575, out. 2003.

BARBOSA, R. M. et al . Aborto induzido entre mulheres em idade reprodutiva vivendo e não vivendo com HIV/aids no Brasil. Revista Ciência \&. Saúde Coletiva, Rio de Janeiro, v.14, n. 4, p.10851099, ago. 2009.

BARDIN, L. Análise de conteúdo. Lisboa : Edições 70, 2009, 221p.

BENUTE, G. R. et al. Abortamento espontâneo e provocado: ansiedade, depressão e culpa. Revista da Associação Médica Brasileira, São Paulo, v. 55, n. 3, p. 322-327, jan. 2009.

BOEMER, M. R.; MARIUTTI, M. G. A mulher em situação de abortamento: um enfoque existencial. Revista da Escola de Enfermagem da USP, São Paulo, v. 37, n. 2, p. 59-71, 2003.

BRASIL. Ministério da Saúde. Conselho Nacional de Saúde. Comissão Nacional de Ética em Pesquisa. Resolução n. 196, de 10 de outubro de 1996. Dispõe sobre pesquisa envolvendo seres humanos. Brasília, 1996. Disponível em: <http:// conselho.saude.gov.br/comissao/conep/resolucao. html> Acesso em: 10 jun. 2009.

BRASIL. Ministério da Saúde. Aborto e saúde pública no Brasil: 20 anos. Brasília, DF, 2009. Disponível em <http://bvsms.saude.gov.br/bvs/ publicacoes/livro_aborto.pdf >. Acesso em: 18 mar. 2009. 
BRASIL. Ministério da Saúde. Secretaria de Atenção à Saúde. Departamento de Ações Programáticas Estratégicas. Atenção Humanizada ao Abortamento: norma técnica Brasília, DF: Ministério da Saúde, 2005. Disponível em <http://portal.saude.gov.br/portal/ arquivos/pdf/atencao_humanizada.pdf $>$. Acesso em: 25 mar. 2009.

CECATTI, J. G., et. al. Aborto no Brasil: um enfoque demográfico. Revista. Brasileira de. Ginecologia e Obstetrícia. Rio de Janeiro, v. 32, n. 3, mar. 2010.

CHUMPITAZ, V. A. Percepções femininas sobre a participação do parceiro nas decisões reprodutivas e no aborto induzido. 2003. $140 \mathrm{f}$. Dissertação (Mestrado em Ciências na área de Saúde Pública) - Fundação Oswaldo Cruz, Escola Nacional de Saúde Pública, Rio de Janeiro, 2003.

GESTEIRA, S. M. A. Assistência prestada à mulher em processo de abortamento provocado: o discurso das mulheres e das profissionais de enfermagem. 2006. 229f. Tese (Doutorado em Enfermagem) - Escola Paulista de Medicina, Universidade Federal de São Paulo, São Paulo, 2006.

GESTEIRA, S. M. A.; BARBOSA, V. L.; ENDO, P. C. O luto no processo de aborto provocado. Acta Paulista. de Enfermagem. São Paulo, v. 19, n. 4, p. 462-467, out./dez. 2006.

MARIUTTI, M. G.; FUREGATO, A. R. F. Fatores protetores e de risco para depressão da mulher após o aborto. Revista Brasileira de Enfermagem, Brasília, v. 63, n. 2, abr. 2010.

NADER, P. R. et al.. Características de abortamentos atendidos em uma maternidade pública do Município da Serra- ES. Revista. Brasileira de Epidemiologia. São Paulo, v.1o, n.4, p. 615-624, dez. 2007.

ORGANIZAÇÃO DAS NAÇÕES UNIDAS.

Convenção para eliminação de todas as formas de discriminação contra mulher. CEDAW, General Recommendation 24, Women and Health (art. 12). Estados Unidos, 1999. Disponível em <www. pge.sp.gov.br/centrodeestudos/bibliotecavirtual/ instrumentos/discrimulher.htm>. Acesso em: 25 mar. 2009.
PÉREZ, B. A. G. Aborto provocado: representações sociais de mulheres. 2006.130 f. Dissertação (Mestrado em Enfermagem) - Escola de Enfermagem, Universidade Federal da Bahia, Salvador, 2006.

RAMOS, K. S.; FERREIRA, A. L. C. G.; SOUZA, A. I. Mulheres hospitalizadas por abortamento em uma Maternidade Escola na Cidade do Recife, Brasil. Revista daEscola de Enfermagem da USP, São Paulo, v. 44, n. 3, set. 2010.

SANDI, S.F.; BRAZ, M. As mulheres brasileiras e o aborto: uma abordagem bioética na saúde pública. Revista Bioética, Brasília, v. 18, n. 1, jun. 2010.

SCAVONE, L.; CORTES, G. Do subjetivo ao social: implicações sociológicas do aborto. In: Scavone, L. \& Batista, L.E. (Org.). Pesquisa de gênero: entre o público e o privado. Araraquara: Faculdade de Ciências e Letras, Laboratório Editorial, 200o. p. 27-48.

SILVA, D. et al.. Aborto provocado: redução da frequência e gravidade das complicações. Consequência do uso de misoprostol. Revista BrasileiradeSaúde Materno Infantil. Recife, v.10 n..4, out./dec. 2010.

SILVA, R. S.; VIEIRA, E. M. Frequency and characteristics of induced abortion among married and single women in São Paulo, Brazil. Cadernos de Saúde Pública, Rio de Janeiro, v. 25, n. 1, jan. 2009.

SOUZA, V.L.C. A violência conjugal e suas influências na decisão da mulher pelo aborto. 200o. 147 p. Dissertação (Mestrado em Enfermagem) - Escola de Enfermagem , Universidade Federal da Bahia, Salvador.

Recebido em: 29/04/20II Reapresentado em: 03/02/2012 Aprovado em: 10/09/2012 